\title{
Remote supply
}

\section{KEY POINTS}

n The requirements for remote supply of veterinary medicines are the same as for face to face supply

- Prescribers and dispensers must be aware of prescription misuse and the ways of mitigating it

- Care must be taken when dispatching medications by post or courier to ensure that they reach the correct recipient without damage

- Returned veterinary medicines may not be returned to stock for resale, but protocols must be in place to avoid a legal obligation to refund clients if purchases are returned under distance selling laws

- Advertising of prescription-only medicines to the general public is an offence

- Internet retailers can apply for accreditation under the Veterinary Medicines Directorate (VMD)'s Accredited Internet Retailer Scheme (AIRS), which provides reassurance to customers

UK law permits the remote supply of all categories of veterinary medicinal products (VMP), provided the legal requirements regarding prescription and supply are met. The requirements are the same regardless of whether a transaction is online or face to face.

Remote supply is commonly associated with internet sites (@). Although these are often informally described as 'internet pharmacies', this usage should be discouraged by staff if the business is not a retail pharmacy registered with the General Pharmaceutical Council (GPhC) in Great Britain or the Pharmaceutical Society of Northern Ireland (PSNI), as the term 'pharmacy' is a restricted title. Inappropriate use in connection to a business may result in legal action from the GPhC or PSNI. Some internet sites are true pharmacies controlled by pharmacists, and a number of sites are under the control of suitably qualified persons (SQPS), but many are hosted by veterinary practices or other organizations under the professional control of veterinary surgeons (veterinarians) and are therefore best referred to as internet or online retailers.

As well as online ordering, some businesses also promote mail order supply of VMP through more traditional printed price lists and advertisements, and others (including many veterinary practices with clients in more remote areas) may wish to supply medicines by post or courier on an ad hoc basis as an occasional customer service.

\section{Legislative requirements}

The general requirements of UK legislation apply to the prescription and supply of medicines, irrespective of whether a client physically visits the premises and meets the veterinary surgeon (or pharmacist/SQP) face to face. A veterinary surgeon supplying medicines remotely must be able to demonstrate that they comply with the Veterinary Medicines Regulations (VMR), including the supply, registration, storage and inspection requirements. The same regulation applies to SQPs overseeing remote supply of appropriate products to clients (as many veterinary practices employ SQPs for dispensary duties).

As a result, although the supply of VMP ordered online or via direct mail can be carried out legally, veterinary medicines (other than those classified as 'authorized veterinary medicine - general sales list' (AVM-GSL) and Exemption for Small Pet Animals (ESPA) medicines) should not be offered or supplied via auctions (such as eBay or Gumtree), since legal and professional obligations cannot be met satisfactorily.

Veterinary premises that supply medicines ordered online or via direct mail under the professional control of a veterinary surgeon must be registered as veterinary practice premises and inspected either by the VMD or a Royal College of Veterinary Surgeons (RCVS) Practice Standards Scheme (PSS) inspector.

The VMD publishes guidance, including the supply of medicines remotely, on the retail of veterinary medicines webpage (@).

\section{Prescriptions}

The requirements for the prescribing and supplying of VMP are the same for remote supply as for face to face. However, key points to bear in mind include:

- Veterinary surgeons must ensure that they have sufficient information to make a clinical judgement about the animal and the correct medicine to prescribe

- Prescription-only medicine - veterinarian (POM-V) medicines may only be prescribed for animals under the veterinary surgeon's care 
- Prescription-only medicine - veterinarian, pharmacist, SQP (POM-VPS) and Non-food animal - veterinarian, pharmacist, SQP (NFA-VPS) medicines may be prescribed or supplied for animals not under the veterinary surgeon's care, but the other professional and legal obligations must be met

- Where POM-VPS or NFA-VPS medicines are being prescribed or supplied by a pharmacist or SQP, the prescriber's professional and legal obligations likewise remain unchanged in terms of selecting a medication that is suitable for the animal(s) and the client.

See also Prescribing, supplying, dispensing and labelling procedures.

\section{Dispensing against prescriptions}

Prescriptions may be faxed or emailed to an internet or mail order supplier. Electronic transmission of prescriptions for Controlled Drugs (CDs) in Schedules 2 and 3 of the Misuse of Drugs Regulations is not allowed. Note that the drugs present in these Schedules do change periodically (for example, the inclusion of gabapentin and pregabalin in Schedule 3 from April 2019) and so up-to-date knowledge of drug classifications, especially for human medicines prescribed under the cascade, must be maintained. It is incumbent upon the dispensing professional (in this case, almost invariably the veterinary surgeon) to ensure that they are operating in accordance with the law as it stands when the drug is dispensed, which may in rare cases have changed since the medication was prescribed. A suitable source of reference should therefore be sought in situations where the dispensing professional is unfamiliar with the drug prescribed (@)).

Prescriptions for VMP must contain all of the information required by law in order to be valid. There are additional requirements for prescriptions for Schedules 2 and 3 CDs.

\section{(9) See also Controlled Drugs.}

Suppliers have a legal obligation to take all reasonable steps to ensure that a prescription is genuine, is issued by a person entitled to prescribe and is only fulfilled once (unless it is repeatable, in which case the number of repeats must be stated on the prescription). Whatever method is used to ensure the validity of received prescriptions, it is important to be able to demonstrate that these steps have been taken, in case of audit or investigation by the VMD.

Prescription misuse is a growing concern and veterinary surgeons should take care when writing and dispensing against written prescriptions. Common examples include the same prescription being used simultaneously at multiple retailers or counterfeiting the prescriber's authorization (considered to be fraud), and amendments to the date or quantity specified on a prescription (considered a lesser offence by the VMD if for a non-food animal, but still illegal). If it is unclear whether prescription fraud or misuse has occurred, it is good practice for the dispensing professional to contact the prescriber directly to confirm the validity of the prescription and any alterations or amendments. If a veterinary surgeon suspects that a prescription has been misused, it should be reported to the VMD via its prescription misuse reporting form available on the VMD's website (@). Guidance on how to mitigate prescription misuse is also available on the Retail of veterinary medicines page (O)).

Veterinary surgeons providing a written prescription should seek to ensure that it will be legally filled. The VMD encourages prescribers to include text on their prescriptions raising awareness of its Accredited Internet Retailer Scheme (AIRS) and the importance of sourcing medicines from responsible sources. The VMD will provide suitable text and a copy of its AIRS logo to include on prescriptions upon request.

You may only buy these prescribed medicines from another veterinary practice or a pharmacy. If you buy them online, we recommend that you use a Veterinary Medicines Directorate (VMD) accredited internet retailer. VMD accredited internet retailers will display their AIRS logo containing their unique accreditation number on their website. Clicking on the logo will confirm their accreditation details on the VMD's website (@)).

\section{Supply by post}

Veterinary surgeons may legally supply medicines by post or courier, whether operating from a traditional veterinary practice, an internet site or a mail order service.

Veterinary surgeons should take account of whether the VMP are potentially harmful to the general public. Medicines not in the manufacturer's packaging should be supplied in child-resistant containers. Appropriate safeguards should be taken to protect the medicine in transit; for example, medicines that are in liquid form will require different safeguards from those that must be kept refrigerated. The Royal Mail (@) provides guidance on what can and cannot be sent in the post. Be aware that the regulations for business use are not necessarily identical to those for personal mailings. Note also that some topical preparations may contain a flammable liquid base, in which case it is necessary to comply with the requirements for flammable liquids. If a courier is to be used, it is necessary to confirm that they will accept medicines and/or flammable liquids, as some may refuse to carry such products. In many cases, products sent that are in breach of the company's terms and conditions will be destroyed if detected.

If packages are reported lost in transit, with no evidence as to whether this is in fact the case, it is difficult for the dispensing professional to refuse to re-dispense, even though this would (if the client was misleading them) strictly amount to prescription fraud by the client. Some mechanism should be in place to minimize this risk, for example, by dispatching any POM-V products by a recorded and signed-for delivery route. By doing so, it is possible to demonstrate, if needed, whether the product has been delivered or genuinely lost in transit. This would also assist the supplier in meeting their legal requirement under the VMR to 'take all reasonable steps to ensure that it is supplied to the person named in the prescription' and is therefore suggested as best practice.

If cold-chain medications are to be dispatched, it is necessary to ensure that cold chain will be maintained throughout the dispatch, processing and delivery process. It is considered good practice to be able to demonstrate that the chosen packing, insulation or system will maintain products at $+2-+8$ degrees throughout by, for example, dispatching a sample package containing a data logger or max-min thermometer, and recording the results on receipt. 
In general, CDs should not be sent via post, but if this is essential then they should be sent at least by recorded delivery to ensure an audit trail, and preferably via a service that ensures the CDs are only handed over to a competent adult.

The standard legal obligations on suppliers apply, including:

neing satisfied that the person who will use the VMP is competent to do so safely, and intends to use it for its authorized purpose

- Advising on safe administration and on any necessary warnings or contraindications on the label or package leaflet

- Supplying only the VMP named on the prescription; unlike in human pharmacy, generic substitution is not permitted. Where the prescription specifies a generic name rather than an identified VMP, only authorized products (not human medications, 'specials' or products imported under an SIC) can be supplied, unless the prescription states otherwise.

\section{Returns}

See also Correct storage of medicines, dispensary management and standard operating procedures.

Medicines that have been dispensed and dispatched may not be returned to stock for resale if the storage conditions cannot be guaranteed. This is likely to be the case for all remotely supplied medicines dispatched by post or courier (with the possible exception of cold-chain products returned to the dispatcher undelivered). However, under UK law (The Consumer Contracts (Information, Cancellation and Additional Charges) Regulations 2013 (@), clients are entitled to an automatic refund on return of undamaged products sold unseen ('distance selling').

This does not apply to prescription medicines (POM-V and POM-VPS), which have a specific exemption from the Part 3 Right to Cancel (para. 27 (2)). However, NFA-VPS and AVM-GSL medications are not strictly covered by this exemption, as they are not prescription medicines. In some situations, this could result in retailers being forced to refund the customer and destroy the product as pharmaceutical waste.

Other exemptions which could be held to apply would include orders under $£ 42$ (para. 27 (3)); 'the supply of goods which are liable to deteriorate or expire rapidly' (para. 28 (1) c); or the 'spoilage' clause at 34 (9). However, for either of these latter to be considered a defence, the customer should be made aware that the retailer takes this interpretation before purchase.

Where products are to be dispensed, supplied or dispatched remotely, it is advisable to ensure that your terms of business with your clients specify what exemptions to returns are being used, and that the right of cancellation and return does not apply to these products. Note that where other, non-medicinal, products are also being sold, the right to cancel still applies to that component of any order.

\section{Examples}

The following examples demonstrate some of the ways in which the requirements at the time of supply of VMP can be met, with particular reference for internet and mail order retailers.

- It is considered good practice for all businesses supplying VMP to clearly display the authorization details (e.g. name and registered number) of the veterinary surgeon, pharmacist or SQP responsible. This person should be available to advise clients directly.

- It must be possible for a client to be given direct advice so that the most appropriate medicine is prescribed/supplied to them, regardless of the medicines that the supplier has in stock (or any special offers).

- Even if a client asks for a specific POM-VPS or NFA-VPS medicine, there must be an interaction between the client and supplier to ensure that it is the appropriate medicine for the animal and circumstances (including husbandry and condition). The use of customer disclaimers (such as 'yes'/'no' tick boxes) and simple 'add to basket' with no customer interaction is not acceptable.

- For clients who wish to order POM-VPS or NFA-VPS medicines over the internet, an online registration system should be set up so that details of the client and of the type, number, weight, age and other details of their animals are recorded, up to date and can be used to enable a supplier to make the necessary checks on suitability of the medicine ordered before any are supplied. The batch number and expiry date of products dispensed should be recorded to enable a batch recall to be enacted quickly and accurately from the end user. This would also enable returning customers to log in without having to provide this information again, unless it has changed, and there should be a confirmatory declaration with each order to this effect

- Internet suppliers may also set up an online questionnaire for clients to confirm whether they have administered the VMP previously, if they are aware of the relevant safety precautions and to confirm that they will read the packaging and product literature before using the medicine. This does not, however, absolve the dispensing professional of their legal responsibilities to prescribe or supply the most appropriate product for the animal(s) in question and should be considered an adjunct to collecting the data above rather than a replacement for it

- An email or telephone call may be made to the client following order placement, to enable the supplier to discuss any problems before supplying the medicine. This approach would be considered good practice and must happen if there is any missing or conflicting information, or if the dispensing professional believes the product selected by the client is unsuitable.

- All information provided must be carefully checked by the dispensing professional before any supply is made.

- Records of communication with clients should be made and retained.

- All records relating to the client may comprise or contain personal data, and therefore the General Data Protection Regulation (GDPR) applies. In an online retail environment, additional security protocols must be instituted to protect client data, which is relatively easily accessible compared with that stored on a hardened Practice Management System (PMS). For more information, see the Information Commissioner's Office guidance (@). 


\section{Advertising}

Advertising of POM-V and POM-VPS medicines is only permitted to defined groups, which do not include pet owners, clients or the general public. Following a change in law in 2013, antimicrobial VMP cannot be advertised to professional keepers of animals. In addition, POM-VPS medicines cannot be advertised to non-professional horse owners or keepers.

The VMD define advertising as 'any activity that is aimed or designed to promote the sale, supply or use of a veterinary medicine, including electronic advertising (for example website banners, emails), but not including price lists'. The home page of a website is deemed to be a public space, and therefore, no POM-VPS or POM-V products should be presented there; likewise, price promotions (such as 'special offer' or 'buy one get one free') and social promotions ('people who bought $X$ commonly buy $Y^{\prime}$ ) are considered advertising and should not be applied to these classes of drugs.

However, price lists (printed or online) of POM-V or POM-VPS medicines may be supplied to the general public, provided certain conditions are met:

- All products within a particular category must be included (e.g. all 'flea treatments' or all 'anti-inflammatory drugs' stocked by the retailer)

- Text and images displayed must all be of the same size and type; it is unacceptable for a single medicine on a price list to feature more prominently than the rest

- The name of each medicine, its image and a description may be shown within a price list, providing that the wording is in accordance with the VMP's published summary of product characteristics (SPC). The name of the medicine should be exactly as its full authorized name. This is important, as different medicines within the same brand should be clearly distinguished

- A description may be given (e.g. 'dog flea treatment') as long as it is in accordance with the SPC

- Any image of the packaging used must show the UK authorized packaging

- Pay-per-click advertising (paid for advertisements on search engines) is deemed to be a price list as long as the main keyword used is the brand name of the medication, as under these circumstances the VMD's interpretation is that clients will not see a product unless they have specifically searched for it.

There are no specific restrictions placed upon NFA-VPS and AVM-GSL medications, provided that any advertising contains no misleading information, and that the claims that are made for the VMP are in accordance with its SPC.

It is not lawful to advertise, or even to include in price lists, products whose only legal use is under the cascade (i.e. human authorized medications, 'specials', and imported medicines). These can legally be sold by retail supply, if in receipt of a suitable prescription, but not displayed openly on a website or price list. A statement to the effect that: 'If you have a prescription from your vet for a medication that is not listed, please contact us' is legal, and is a useful adjunct to a website or mail order price list if these products are stocked.

The VMD publishes guidance on the advertising of VMP (@)

\section{Non-UK websites}

UK law requires that (save for the exemptions provided to veterinary surgeons under the cascade) only authorized VMP should be used.

It is an offence for an animal owner to:

- Be in possession of a VMP not lawfully supplied in the UK

- Administer a VMP unless it has a marketing authorization valid in the UK or comes under the ESPA

- Import a VMP into the UK, even if authorized for use in the UK (except for AVM-GSL and ESPA medicines)

- Supply a VMP to another person, other than as legally required.

It may be helpful for veterinary surgeons to ensure that animal owners requesting a written prescription, or otherwise intending to source medicines via the internet, are aware of the importance of a UK-based and legal supplier. Only by doing so can owners be sure that their animals will receive safe and effective medicines - and avoid breaking the law themselves. Illegally sourced medicine may be counterfeit, ineffective or unsafe for the client's animals.

\section{Internet retailers}

\section{VMD Accredited Internet Retailer Scheme}

Internet retailers of VMP can apply for accreditation under the VMD's Accredited Internet Retailer Scheme (AIRS). Retailers who meet the accreditation criteria will be able to display a logo on their website.

This logo includes the retailer's unique accreditation number. Clicking on the number will take the customer to the retailer's entry in the register of accredited retailers on the VMD website, allowing them to check the accreditation status.

The VMD's AIRS is a means of facilitating self-regulation by UK-based internet

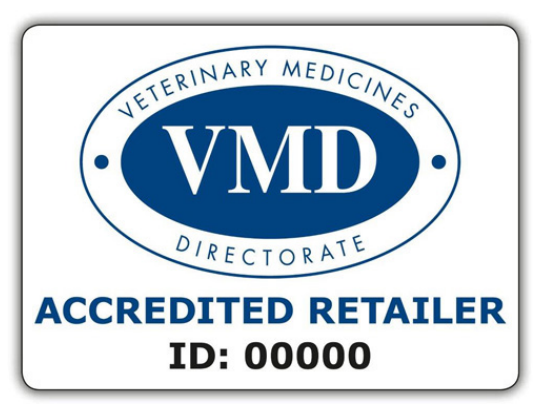
retailers supplying VMP. Following accreditation, onsite inspections of the internet retailer's premises (if they have not been inspected already) will be carried out, to check compliance with the VMR. It is a voluntary scheme and is free of charge.

Further information about the scheme may be found on the VMD website (@).

The VMD have also produced detailed guidance as to the operation of an internet retailer which is available here (@). 


\section{Illegal sales of VMP via the internet}

The VMD's Enforcement Team acts on complaints it receives about internet retailers. When appropriate, the VMD's inspectors or the Department for Environment, Food and Rural Affairs (Defra) Investigation Services will investigate allegations of illegal activities relating to the importation, supply and administration of VMP. If practices are concerned that a client may have been supplied illegal products, they can discreetly report incidences to the enforcement team (see Legal controls on veterinary medicines (@)) or contact the enforcement team for more details on enforcement@vmd.gov.uk.

\section{Useful links}

Royal Pharmaceutical Society (:) Veterinary Medicines Directorate (@) Prescription misuse reporting (@) Veterinary Medicines Guidance (@) VMD Model Retailer Guidelines (:)

The Veterinary Medicines Regulations 2013 (@)

The Consumer Contracts (Information, Cancellation and Additional Charges) Regulations 2013 (@)

\section{QUESTIONS}

1. What prescriptions can be sent electronically to be fulfilled?
a. All
b. Only prescriptions for non-Controlled Drugs
c. Prescriptions for non-Controlled Drugs and Schedule 4 and 5 Controlled Drugs
d. None

2. To whom can you report a suspected prescription misuse?
a. VMD
b. Police
c. VMD and Police
d. The Suspected Adverse Reaction Reporting Scheme

3. How can an internet retailer comply with the requirements to prescribe/supply a POM-VPS medicine?
a. Display a disclaimer saying the customer is responsible for purchasing the correct product
b. Add to basket with a tick box asking the customer to confirm they have read the Terms and Conditions
c. Have a detailed questionnaire that the customer has to complete and which is then assessed by the registered qualified person
d. Require a face-to-face consultation

4. What does the VMD's AIRS scheme do? a. Provides a means for customers to check they are buying from a reputable and appropriate UK supplier of
VMP
b. Check the registered website systems and processes ensure compliance with the law
c. Confirm that the retailer's premises have been inspected
d. All of the above 